$$
\begin{aligned}
& \text { NAL PROPOSAL No. } 66 \\
& \text { Correspondent: } \begin{array}{l}
\text { J. VanderVelde } \\
\text { Physics Department } \\
\text { University of Michigan } \\
\text { Ann Arbor, Mich. 48104 }
\end{array} \\
& \text { FTS/Off-net: } \quad \begin{aligned}
313-769-7100 \\
764-1817
\end{aligned}
\end{aligned}
$$$$
\text { STUDY OF LOW-MASS PERIPHERAL STATES }
$$$$
\text { IN A SMALL TRIGGERED BUBBLE CHAMBER }
$$

J. Chapman, J. Lys, H. Ring, B. Roe, D. Sinclair, J. VanderVelde University of Michigan 


\section{Study of Low-mass Peripheral states in a Small Triggered Bubble Chamber}

I. Abstract

We propose to study $\sim 40,000$ events of the type

$$
\text { beam particle }+ \text { proton } \rightarrow M+\text { beam particle }
$$

where $M$ is a fairly low mass $(2-5 \mathrm{GeV})$ state which is peripherally produced and slow in the lab. The decay products of $M$ would be studied in detail as a function of $M$ at two different beam momenta. The choice of beam particle and the two beam momenta would depend on what was technically most feasible, but at least the beam momenta should be greater than $100 \mathrm{GeV} / \mathrm{c}$.

A hydrogen bubble chamber, pulsing several times per machine cycle, would be triggered on a fast forward particle near the beam momentum. The invariant mass $M$ would be calculated for each event by momentum analysis of this particle.

The experiment requires a beam with a momentum resolution $\Delta \mathrm{p} / \mathrm{p}$ of .002 or better and an emittance of $.1 \mathrm{~mm}$ - mrad.

Experimenters

J. Chapman, J. Lys, H. Ring, B. Roe, D. Sinclair, and J. Vander Velde, Physics Department, The University of Michigan.

Correspondent

J. Vander Velde, Physics Department, The University of Michigan, Ann Arbor, Michigan, 48104. 
II. Physics Justification

The study of detailed properties of low mass, peripheral hadronic states may be the most sensitive way to get at the dynamics of high energy strong interactions. By selecting low mass we tavor low multiplicity, which means there is ample "room" in phase space for the events to distinguish between various theories (or help us find new ones). I.e., we expect that the characteristics of such events will be determined more by dynamics than will those of the high multiplicity events, which may be dominated by some type of generalized phase space restrictions.

For various $M$ values in the range $2-5 \mathrm{GeV}$ we can study the decay of $M$ into $\pi, P, K, \Lambda, \rho, N^{*}$, etc. In general we will not be able to detect slow missing neutral particles ( $\leq I \mathrm{GeV} / \mathrm{c}$ ) and we will not be measuring $t$, but summing over it. The dependence on beam momentum may be particularly important in distinguishing various theories, hence our desire to have two similar exposures at two different momenta.

Technically, the principle advantages of the experiment are these:

(I) We select only that fraction (10-30\%) of the cross section which is due to very peripheral events.

(2) The value of the excitation mass $M$ is determined for each event independently of its decay contiguration in the bubble chamber so that the $M$ dependence of the decay properties can be determined.

(3) The state $M$ is restricted to low momentum $(2-13 \mathrm{GeV} / \mathrm{c})$ in the lab so that our preselection gives events that are amenable to measurement in the chamber.

(4) The triggering scheme will insure that there are no 
missing neutrals of momentum $>13 \mathrm{GeV} / \mathrm{c}$, so that we are strongly biased in favor of single dissociation type events.

Note that we are not trying to measure do/dMdt, which can be done with counters; but are interested in the internal properties of the state $M$ itself.

III. Equipment and Technical Details

We give below a description of the necessary equipment, and some calculations showing the feasibility of the method. The specific calculations are for $P_{0}=200 \mathrm{GeV} / \mathrm{c}$.

BEAM: Protons on pions with momentum $>100 \mathrm{GeV} / \mathrm{c}$ $\frac{\Delta p}{p}= \pm .001$ or better

Horizontal emittance $1.0 \mathrm{~mm}-\mathrm{m}$ rad

Vertical emittance $5.0 \mathrm{~mm}$ - $\mathrm{m}$ rad

Horizontal focus in chamber $\sim 1 \mathrm{~mm}$

Vertical dimension in chamber $\sim 3$ inches converging to a focus $\sim 20$ meters downstream from the chamber.

CHAMBER:

Hydrogen bubble chamber capable of pulsing at least 5 times per machine cycle. Lights need only fire once per pulse. $\sim 10$ tracks per expansion and 5 expansions per pulse give $~ 75$ feet of track per pulse in a $1.5 \mathrm{ft}$. fiducial volume.

SPECTROMETER:

A focussing doublet placed just upstream of the bending magnet makes a horizontal image of the chamber focus on a counter array $~ 50$ meters downstream. In the vertical plane this doublet acts as a field lens. Quadrapole dimensions 4" $772^{\prime \prime}$. 
Bending magnet $4^{\prime \prime} \times 6^{\prime \prime} \times 20 \mathrm{ft}$. Iong with $20 \mathrm{~K}$. gauss field.

\section{KINEMATICS AND RESOLUTION}

(I) $-t=2 m_{p}\left(E-E_{0}\right)-\left(M^{2}-m_{p}^{2}\right) \approx P_{\perp}^{2} \approx 0$

$-t_{\text {min }} \approx\left(\frac{M^{2}-m_{b e a m}^{2}}{2 P_{0}}\right)^{2}$ is negligible.

$\Delta \mathrm{M}^{2}$ bite of each counter determined from

$$
\Delta\left(M^{2}-m_{p}^{2}\right) \approx 2 m_{p} \Delta E
$$

(2) $\triangle E$ determined by angular resolution of spectrometer.

$$
\Delta \theta_{\text {rès }}=\frac{\text { object size }}{\text { upstream focal length }}=\frac{.0015}{15}=10^{-4} \mathrm{rad} \text {. }
$$

abend of (for example) $200 \mathrm{GeV} / \mathrm{c}$ particle in $400 \mathrm{~K}$ gaussfeet $=.02 \mathrm{rad}$.

gives $\Delta E=\frac{\Delta \theta}{\theta}=200 \cdot \frac{\left(10^{-4}\right)}{.02}=1 \mathrm{GeV}$.

So $\Delta \mathrm{M}^{2} \approx 2 \mathrm{GeV}^{2}$ is the resolution of the spectrometer at $200 \mathrm{GeV} / \mathrm{c}$ which gives $\Delta M=\frac{1}{M} \mathrm{GeV}$. The resolution is proportionally better at lower momentum.

Example of $\Delta M$ bites at $200 \mathrm{GeV} / \mathrm{c}$ :

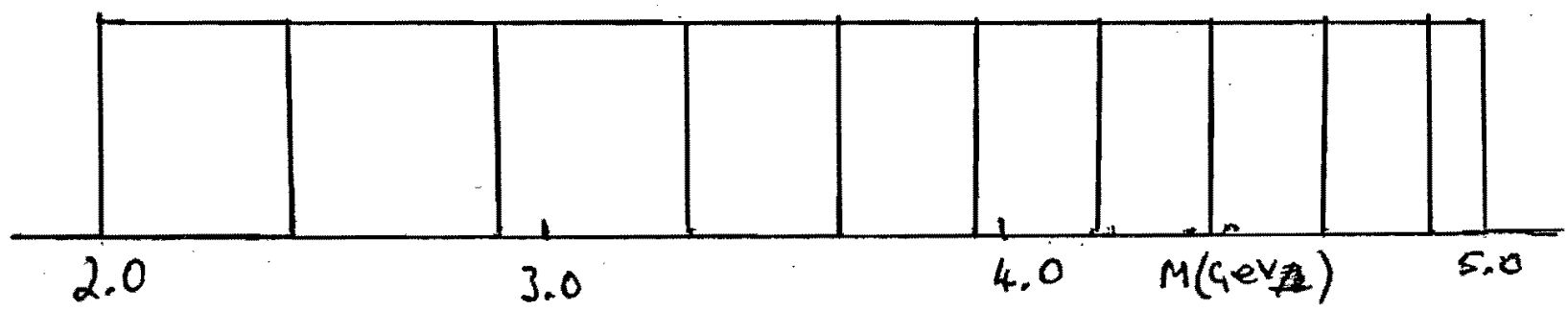

(3) We will accept $-t \approx P_{\perp}^{2}$ up to $\approx 0.25(\mathrm{GeV} / \mathrm{c})^{2}$. At a given $\mathrm{M}^{2}$ the error in $\mathrm{M}^{2}$ introduced by non-zero $\mathrm{P}_{\perp}$ is given by $\delta \mathrm{M}^{2} \approx \mathrm{P}_{\perp}^{2}$. This is negligible due to the exp $\left(-3 \mathrm{P}_{\perp}^{2}\right)$ dependence of cross section, i.e., the distribution of $\delta \mathrm{M}^{2}$

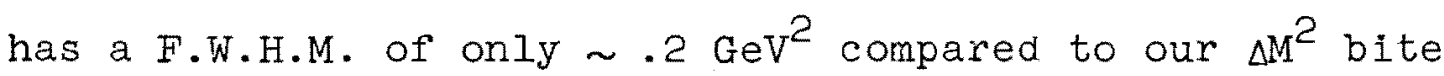
of $2 \mathrm{GeV}^{2}$.

(4) Multiple scattering errors. 
(a) Since the chamber is at a horizontal focus, multiple scattering in the chamber and its walls does not affect the momentum resolution of the chamber. Sill ofrom thet)

(b) In $200 \mathrm{ft}$. of air:

$\delta \theta=3.5 \times 10^{-5}$

(This will be reduced to a negligible value by helium bags, which are also necessary to reduce false triggers.)

(c) Energy loss by ionization in chamber and air amounts to $\sim 30 \mathrm{MeV}$ which is negligible.

EVENT RATE

We estimate from present data that the $M$ interval $2-5 \mathrm{GeV}$ will include $\geqslant 3 \mathrm{mb}$ of cross section. $3 \mathrm{mb}$ means 330 feet of hydrogen per event. With this cross section we get one good event in the fiducial region per expansions or one per 4.5 machine pulses if we can expand times per pulse. About $1.35 \times 10^{6}$ expansions, or 60,000 triggers, will be required for 40,000 events in the $f i-$ ducial region. Thus a considerable saving in running time can be achieved with 5 expansions per pulse. BACKGROUND

We will be triggering on forward particles with momentum in the range $1870198 \mathrm{GeV} / \mathrm{c}$ if we run with a $\lambda$ beam momentum of 200 $\mathrm{GeV} / \mathrm{C}$ (say). If the beam particle is a proton, we expect "background" protons in this range due to the decay of low-mass $\mathrm{N}^{*}$ states which are peripherally produced and have momentum $\sim 200$ $\mathrm{GeV} / \mathrm{c}$. There might be $\sim 10 \mathrm{mb}$ worth of such events, about half of which would give protons as a decay product. If we assume 
that the deacys are predominantly into $\mathrm{p} \pi^{\circ}$ and are isotropic, then about $6-10 \%$ of such protons will have momentum $>187 \mathrm{GeV} / \mathrm{c}$. Thus we expect at most $\sim .5 \mathrm{mb}$ worth of such background protons compared to $\sim 3 \mathrm{mb}$ of "signal" in our triggering. These background events will often be accompanied by a single slow proton in the chamber and an undetected $\pi^{\circ}$ of momentum $\leqslant 13 \mathrm{GeV} / \mathrm{c}$.

The false triggers from interactions in $200 \mathrm{ft}$. of air could be troublesome so the region between the chamber and the counters should be filled with helium.

This experiment is considerably more complicated technically than our other proposal "Study of Multiparticle Production in a Small Bubble Chamber." We believe, however, that the information provided by the spectrometer will be sufficiently valuable to warrant the trouble. The spectrometer provides a measurement of $M$ and also insures that there are no fast missing neutrals. We will be able to detect a missing longitudinal momentum of $\sim$ GeV/c by combining the spectrometer and bubble chamber measure: ments. This is the basic justification for the spectrometer. 